\title{
Generation of 3D synthetic breast tissue
}

\author{
Premkumar Elangovan*a ${ }^{\text {a }}$ David R Dance ${ }^{b, c}$, Kenneth C Young ${ }^{b, c}, K_{\text {Kevin Wells }}{ }^{\mathrm{a}}$ \\ ${ }^{a}$ Centre for Vision, Speech and Signal Processing, University of Surrey, Guildford, GU2 7XH, UK \\ ${ }^{b}$ NCCPM, Royal Surrey County Hospital, Guildford, Surrey, GU2 7XX, UK \\ ${ }^{c}$ Department of Physics, University of Surrey, Guildford, GU2 7XH, UK
}

\begin{abstract}
Virtual clinical trials are an emergent approach for the rapid evaluation and comparison of various breast imaging technologies and techniques using computer-based modeling tools. A fundamental requirement of this approach for mammography is the use of realistic looking breast anatomy in the studies to produce clinically relevant results. In this work, a biologically inspired approach has been used to simulate realistic synthetic breast phantom blocks for use in virtual clinical trials. A variety of high and low frequency features (including Cooper's ligaments, blood vessels and glandular tissue) have been extracted from clinical digital breast tomosynthesis images and used to simulate synthetic breast blocks. The appearance of the phantom blocks was validated by presenting a selection of simulated 2D and DBT images interleaved with real images to a team of experienced readers for rating using an ROC paradigm. The average areas under the curve for 2D and DBT images were $0.53 \pm .04$ and $0.55 \pm .07$ respectively; errors are the standard errors of the mean. The values indicate that the observers had difficulty in differentiating the real images from simulated images. The statistical properties of simulated images of the phantom blocks were evaluated by means of power spectrum analysis. The power spectrum curves for real and simulated images closely match and overlap indicating good agreement.
\end{abstract}

Keywords: virtual clinical trials, breast phantoms, simulated breast texture, synthetic breast model, modelling tools, breast cancer.

\section{INTRODUCTION}

Until recently, 2D-mammography was the gold-standard method for detecting breast cancer in screening owing to good diagnostic performance, short scan time and low radiation dose $^{1}$. Rapid advancements in technology have resulted in the introduction of new modalities into the market. One such example is digital breast tomosynthesis (DBT), in which a series of projections are acquired at limited angles and reconstructed into image planes parallel to the detector. Conventionally, such new technology is evaluated and compared with existing methods using clinical trials with human subjects. However, this process is expensive and requires years to conclude, and slows the pace of technological development. There is an immediate need to supplement physical phantoms and time consuming clinical trials with faster approaches.

Recently, virtual clinical trials (VCTs) have emerged as a complementary tool to conventional clinical trials. VCTs are conducted using computerized modeling tools to simulate normal anatomy and pathology as well as the image formation processes under consideration. In our previous work $^{2}$, we developed and validated a framework for simulating breast imaging by the Hologic Selenia Dimensions 3D system (Hologic, Bedford, Massachusetts, USA). The framework can be easily extended to other 2D and 3D X-ray breast imaging systems. One of the primary requirements of VCTs for mammography is the availability of realistic mathematical models of normal breast anatomy and cancers so that the results are comparable to clinical trials involving patients. In this paper we present a new method of creating realistic 3D mathematical models of breast tissue into which synthetic pathology can be inserted.

For the purposes of simulation, a female breast can be considered as having five major tissue components: (1) adipose tissue; (2) fibro-glandular tissue; (3) Cooper's ligaments; (4) blood vessels and (5) skin. There have been previous attempts to create breast phantoms using mathematical methods ${ }^{3,4}$ and patient $\mathrm{CT}$ data ${ }^{5}$. The mathematically derived 
models tend not to be sufficiently realistic in terms of the breast anatomy. Those based on breast CT images are anatomically correct for large scale features but have lost the high spatial frequencies due to the limited spatial resolution of the imaging system. It therefore remains a challenging problem to produce realistic and detailed breast phantoms. One particular type of virtual clinical trial employs the alternative-forced choice paradigm (2AFC, 4AFC). In this paradigm, only small segments of images of breast tissue are shown to the observers rather than images of the entire breast. This paper presents a biologically inspired approach whereby features/structures extracted from DBT planes of patients are used for constructing realistic phantoms of simulated breast tissue for use in such studies.

\section{MATERIALS AND METHODS}

This section describes how the various breast tissue components were simulated and combined together to produce simulated breast tissue phantoms. The process starts by generating a pseudo-complete breast phantom using an empty skin layer which is then sequentially filled with voxels labelled with different tissue classes. At this stage we have not attempted to replicate realistic global anatomy, but instead aim for realism of local features in small areas of images. Images of the breast model were limited to segments of size $3 \mathrm{~cm} \times 3 \mathrm{~cm}$ or $4 \mathrm{~cm} \times 4 \mathrm{~cm}$.

\subsection{Breast outline and skin layers}

Outlines of compressed breasts were first extracted from a range of DBT images by fitting a 3D mesh on the reconstructed surface. A database of breast outlines was produced by repeating this process on DBT data representing different views (craniocaudal and mediolateral) and different breast thicknesses and sizes. Once the breast outline was established, a $3 \mathrm{~mm}$ tissue layer was added on the surface to simulate the skin layer. Then the rest of the volume was uniformly labeled as adipose tissue.

\subsection{Simulation of glandular tissue}

The shapes of glandular tissue fragments were extracted from DBT images using a region growing method. Initial seed points were selected manually at different locations in the DBT planes and a low tolerance was imposed during the region growing process to avoid segmenting reconstruction artefacts. Subsequently, the extracted fragments were decluttered and de-noised using a series of binary morphological operations. A database of extracted 3D glandular fragments extending approximately $2-3 \mathrm{~cm}$ in each dimension was produced. A combination of visual judgement and previous knowledge of breast structures was used to select suitable glandular tissue fragments from the database.

The selected glandular tissue fragments were shuffled together at random orientations and scale factors and placed inside the fatty breast volume. This process was repeated by adding more glandular fragments to the mix until a particular userdefined glandularity and visual complexity was achieved. The glandular tissue mix was limited to the central $80 \%$ of the breast volume in line with glandular tissue distribution typically found in a real breast. Figure 1.a shows a cross-sectional slice of a breast block produced by combining glandular fragments from the database.

\subsection{Simulation of Cooper's ligaments and blood vessels}

An important component of breast tissue seen with X-ray imaging is the network of linear structures which includes Cooper's ligaments and blood vessels. Therefore, the outlines of Cooper's ligaments and blood vessels were extracted from the DBT planes by a semi-automatic method, performed by manually scrolling though the stack of DBT planes and marking the points at which various linear structures (blood vessels or Cooper's ligaments) were in-focus. 3D spline interpolation was then used to connect these in-focus landmarks to create a 3D wireframe representation of Cooper's ligaments and blood vessels.

The wireframe of linear components was then dilated into cylinders of the desired diameters to render their appearance realistic. The blood vessels were simulated as cylinders of diameter 3-4mm and Cooper's ligaments were simulated as cylinders of diameter 1-2mm. Figure 1.e-f shows an extracted wireframe of Cooper's ligaments and blood vessels after dilation. Finally, the dilated high frequency components were added to the breast volume containing glandular tissue produced in the previous step. When high frequency tissue coincided with glandular tissue, the linear structures replaced the glandular tissue superimposed. Figure 1.b shows the cross-sectional slice of a breast block after addition of linear structures. 


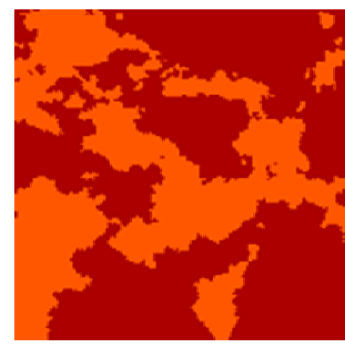

(a)

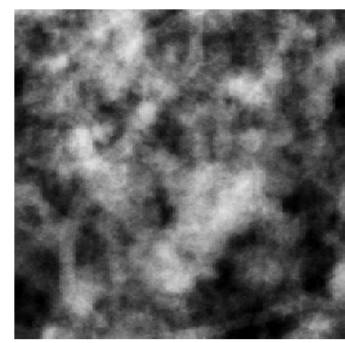

(c)

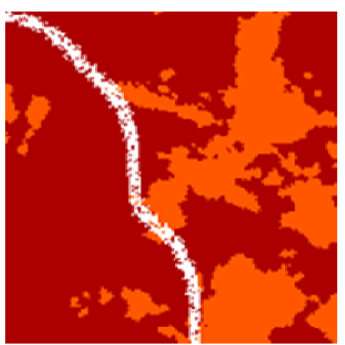

(b)

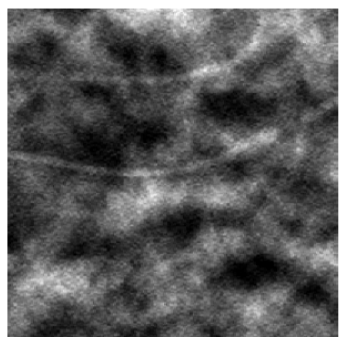

(d)

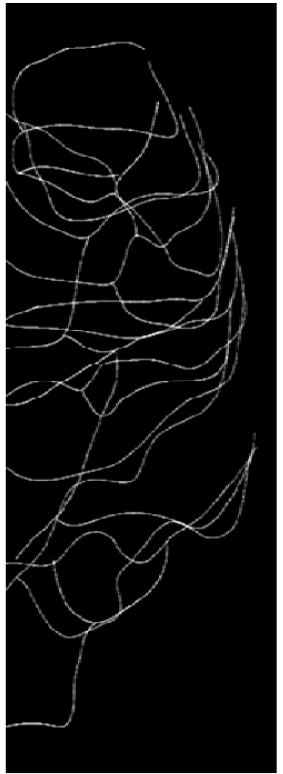

(e)

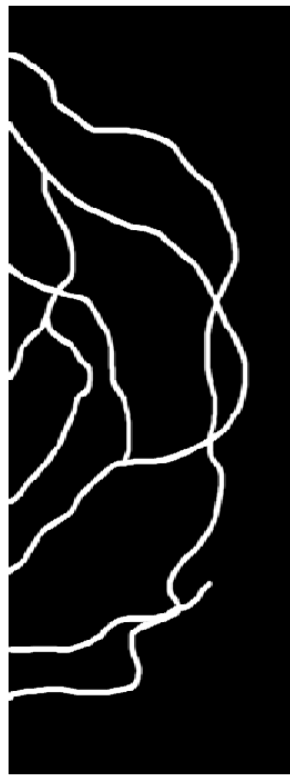

(f)

Figure 1. (a) Cross-sectional slice of a breast block produced by combining various glandular fragments; (b) Cross-sectional slice of a breast block after addition of blood vessels and Cooper's ligaments; (c) processed 2D image (d) and reconstructed DBT plane of a synthetic breast block; (e) representative Cooper's ligaments and (f) blood vessel wireframes extracted from clinical DBT planes of a $6 \mathrm{~cm}$ breast.

\subsection{Image simulation chain}

The whole synthetic breast model was used with a validated image simulation chain ${ }^{2}$ for further evaluation and validation. The image simulation chain is capable of simulating the image acquisition and degradation processes of the Hologic Selenia Dimensions 3D system (Hologic, Bedford, Massachusetts, USA). A brief explanation of the simulation chain is given below. The Hologic system when operating in 'combo' mode produces a 2D image (pixel pitch: 70um) and 15 tomosynthesis projections (pixel pitch: 140um; angular range: $+7.5^{\circ}$ to $-7.5^{\circ}$ ). First, the voxel tissue labels of the breast model were appended with attenuation values derived from the elemental compositions of various breast tissue types (adipose, fibroglandular, skin) published in Hammerstein et al ${ }^{6}$. Subsequently, radiological projections of the breast model were produced using a ray tracing tool for an appropriate set of technical factors. Then, scatter was added to the images using data estimated from Monte Carlo simulations. Experimental measures of MTF (modulation transfer function) and NPS (noise power spectrum) performed on the system being simulated were used to add system blur and noise (system, electronic and quantum) ${ }^{7}$. The mean glandular dose (MGD) was $2.5 \mathrm{mGy}$ for both imaging modalities (2D and DBT). Additional blur was introduced in the simulations to account for the blur due to focal spot size and X-ray tube motion. This simulation process was used to create a 2D projection and 15 tomosynthesis projections. Finally, the unprocessed 2D image was processed using the manufacturer specific image processing tool and the tomosynthesis projections were reconstructed using the manufacturer's filtered back projection algorithm into DBT planes. Figures 1.c and 1.d show the processed 2D image and reconstructed DBT plane of a synthetic breast block phantom respectively.

\subsection{Validation study}

A validation study was conducted by presenting the simulated images to a team of expert observers comprising two specialist radiographers and two consultant radiologists with an average mammographic reading experience of about 15 years. The dataset comprised 35 real and 35 simulated images for each modality (2D and DBT) which were shown in random order to each observer. The observers were asked to rate each of the images on a 6 point scale, 1: Extremely confident that the segment is real, 2: Moderately confident that the segment is real, 3: Slightly confident that the segment is real, 4: Slightly confident that the segment is simulated, 5: Moderately confident that the segment is simulated, 6: Extremely confident that the segment is simulated. The area of the image segments was $3 \mathrm{~cm} \times 3 \mathrm{~cm}$. Images were viewed 
on a high resolution monitor under ideal viewing conditions with no time limitations. These data were then used to construct ROC curves for each observer.

\section{RESULTS \& DISCUSSION}

The average area under the curve (AUC) for 2D and DBT images was calculated to be $0.53 \pm .04$ and $0.55 \pm .07$ respectively; the errors are the standard errors of the mean AUC. The individual ROC plots are shown in figures 2 and 3; the solid diagonal line represents the 50-50 chance line. It can be seen from the 2D ROC plot that the curves cluster around 50-50 chance line indicating that the observers had difficulty in identifying real and simulates images correctly. This trend is consistent with the DBT ROC plot except for one reader (observer 3), an experienced radiologist, whose AUC score was 0.74. Table 1 shows the details of each observer along with the individual AUC values for both modalities. The standard error of the AUC for each observer was computed using the methods of Hanley and McNeil ${ }^{10}$.

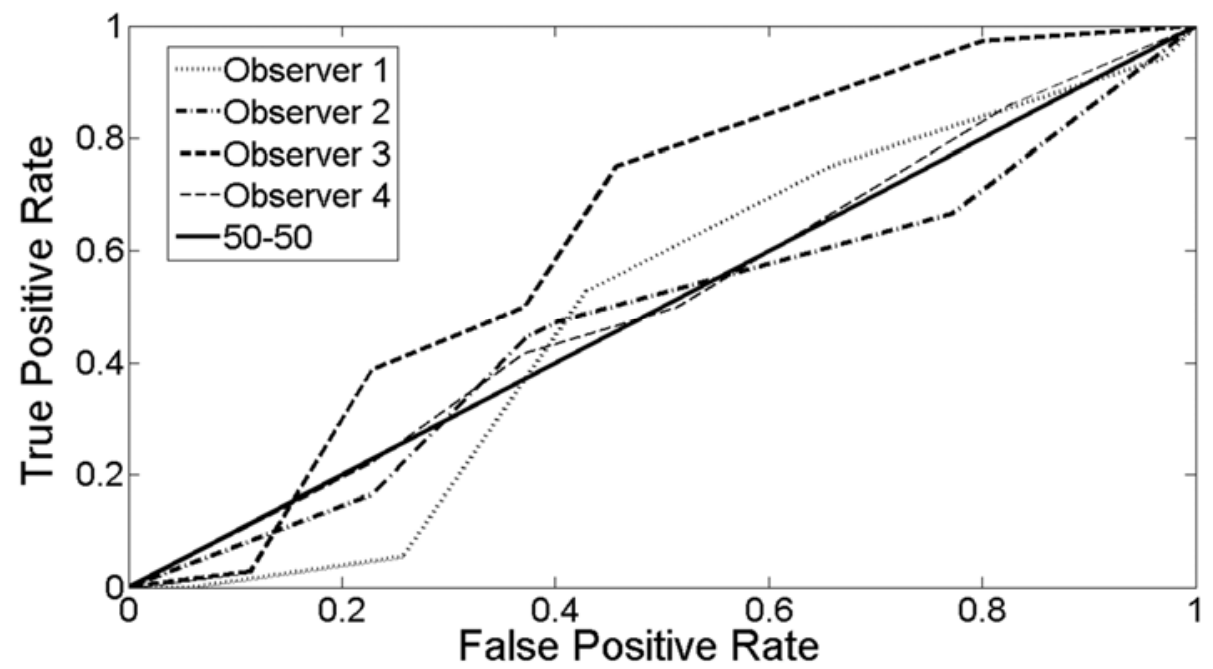

Figure 2. ROC curves for individual observers for 2D images.

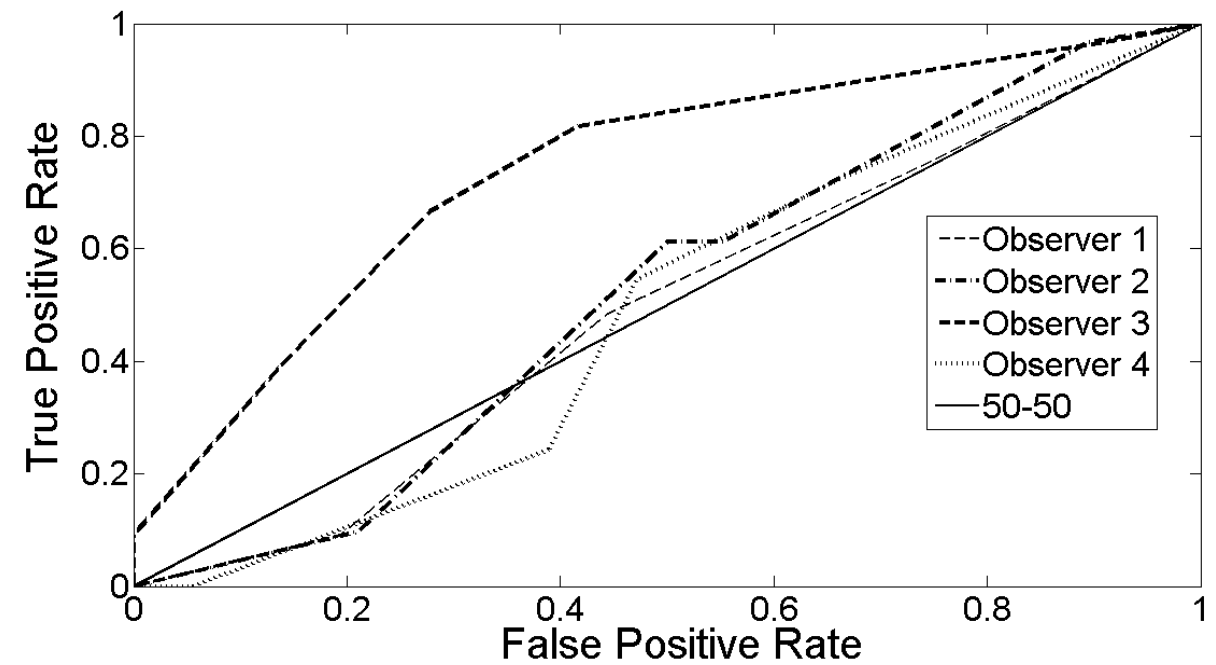

Figure 3. ROC curves for individual observers for DBT images. 
Table 1. AUC values for each modality

\begin{tabular}{|l|l|l|l|}
\hline Observer & $\begin{array}{c}\text { Screening mammography } \\
\text { experience in years }\end{array}$ & AUC (2D) & AUC (DBT) \\
\hline 1 & 18 & $0.50 \pm .08$ & $0.56 \pm .07$ \\
3 & 10 & $0.48 \pm .09$ & $0.52 \pm .04$ \\
4 & $>20$ & $0.64 \pm .09$ & $0.74 \pm .09$ \\
Mean & 14 & $0.51 \pm .07$ & $0.49 \pm .08$ \\
\hline
\end{tabular}

According to the previous literature ${ }^{8}$, structures in a mammogram can be described by a power law spectrum of form $\mathrm{P}(\mathrm{f})=\mathrm{A} / \mathrm{f}^{\mathrm{\beta}}$, where $\beta$ is the power-law exponent and $\mathrm{A}$ is the power spectrum magnitude. Figure 4 shows a comparison of the power spectra of simulated and real 2D image segments (averaged over 300 segments) computed using the method described in Hill et $a l^{8}$ and Cockmartin et $a l^{9}$. The curves for simulated images and real images closely match and overlap indicating good agreement.

The power-law parameters were estimated by applying a linear fit to the log transformed data in the frequency range of $0.2-0.7 \mathrm{~mm}^{-1}$, as this range represents the variations due to breast anatomy ${ }^{8,9}$. The average $\beta$ values for simulated images

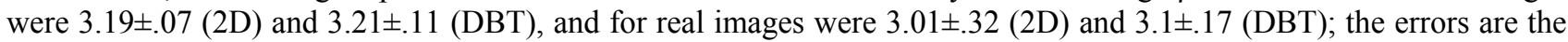
standard errors of the mean $\beta$.

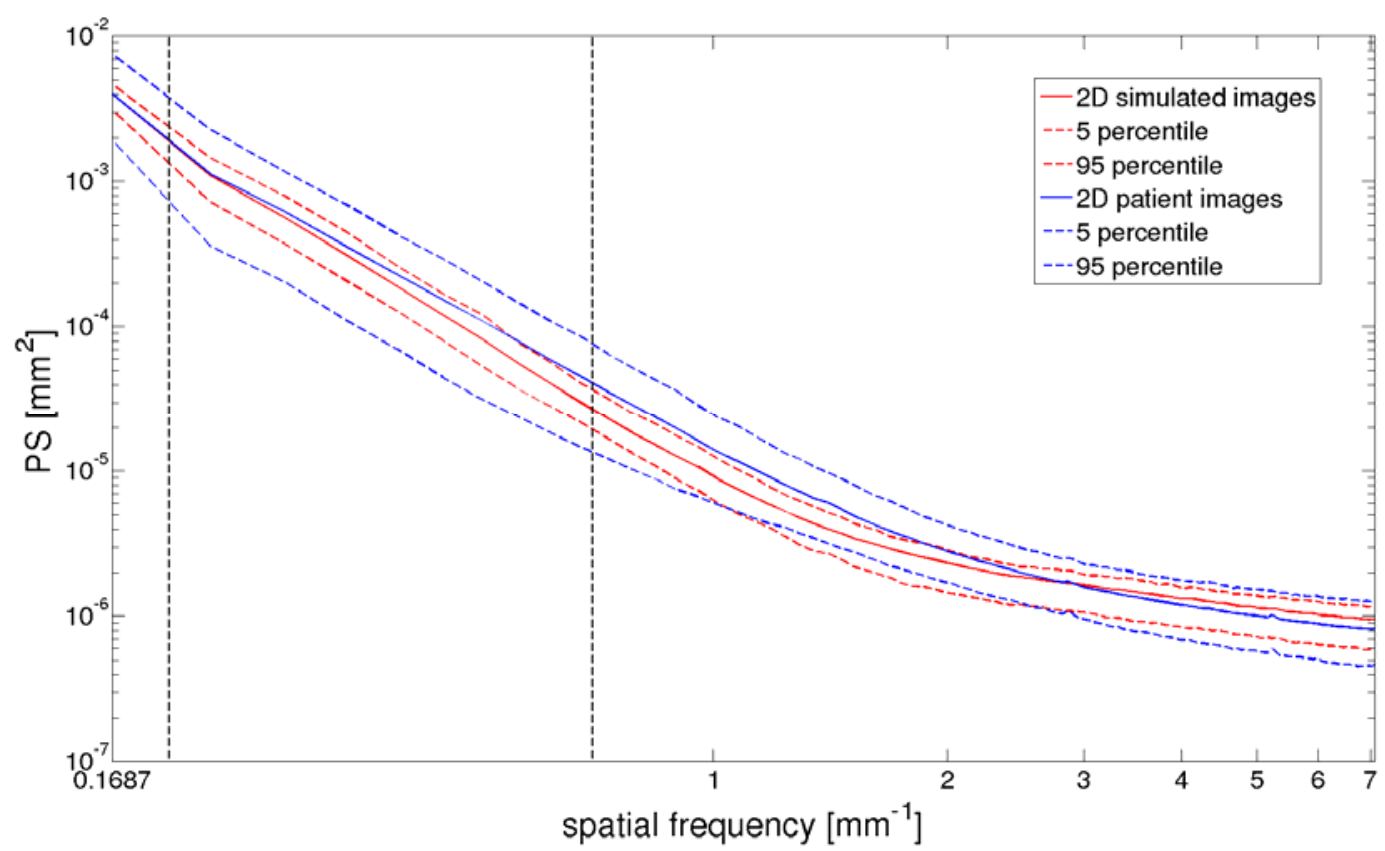

Figure 4. Normalised power spectrum curves for $2 \mathrm{D}$ images.

\section{CONCLUSION}

In this paper, we have described a method for simulating synthetic blocks containing realistic looking breast texture by inheriting anatomical features from real clinical DBT images. The method simulates the components typically visualized in normal breast images, and as a result the appropriate amount of anatomical complexity is represented in the 
radiological images produced from the synthetic blocks. It is clear from the validation study results that the observers had difficulty in differentiating the real images from simulated images. This is the first description of a method of simulating breast tissue by extracting information about the 3D shape of different breast tissue types from DBT images, and the first time an attempt has been made to validate realism of such images using a human observer-based ROC paradigm.

\section{ACKNOWLEDGEMENTS}

This work is part of the OPTIMAM2 project and is funded by Cancer Research UK. We are grateful for Hologic's assistance with the reconstruction. The authors thank colleagues at NCCPM and Dr. Vicky Cooke at the Jarvis Breast Screening Centre, Guildford for invaluable assistance. We also thank Dr Louise Wilkinson, Dr Mamatha Reddy, Charul Patel, Ragini Jhall, Anuma Shrestha, Elizabeth Muscat and Dr Sarah McWilliams from St Georges Hospital, London for participating in the observer study.

\section{REFERENCES}

[1] Hevie, M. A., Chang, J. T., Hendrick, R. E. and Banerjee, M., "Reduction in late-stage breast cancer incidence in the mammography era: Implications for overdiagnosis of invasive cancer," Cancer 120(17), 2649-2656 (2014).

[2] Elangovan, P., Mackenzie, A., Diaz, O., Rashidnasab, A., Dance, D. R., Young, K. C., Warren, L. M., Shaheen, E., Bosmans, H., Bakic, P. R. and Wells, K., "Development and validation of a modelling framework for simulating 2Dmammography and breast tomosynthesis," Phys. Med. Biol. 59(15), 4275-93 (2014).

[3] Bakic, R, B., Zhang, C. and Maidment, A. D. A., "Development and characterization of an anthropomorphic breast software phantom based upon region-growing algorithm," Med. Phys. 38, 3165-3176 (2011).

[4] Bliznakova, K., Bliznakov, Z., Bravou, V., Kolitsi, Z. and Pallikarakis, N., "A three-dimensional breast software phantom for mammography simulation," Phys. Med. Biol. 48, 3699-3719 (2003).

[5] Li, C. M., Segars, W. P., Tourassi, G. D., Boone, J. M. and Dobbins, J. T., "Methodology for generating a 3D computerized breast phantom from empirical data," Med. Phys. 36(7), 3122-3131 (2009).

[6] Hammerstein, G. R., Miller, D. W., White, D. R., Masterson, M. E., Woodard, H. Q. and Laughlin, J. S., “Absorbed radiation dose in mammography," Radiology 130, 485-91 (1979).

[7] Mackenzie, A., Dance, D. R., Diaz, O. and Young, K. C., "Image simulation and a model of noise power spectra across a range of mammographic beam qualities," Med. Phys. 41, 12901-14 (2014).

[8] Hill, M. L., Mainprize, J. G., Carton, A. K., Saab-Puong, S., Iordache, R., Muller, S., Jong, R. A., Dromain, C. and Yaffe, M. J. "Anatomical noise in contrast-enhanced digital mammography. Part I. Single-energy imaging," Med. Phys. 40(8), 051910 (2013).

[9] Cockmartin, L., Bosmans, H. and Marshall, N. W., "Comparative power law analysis of structured breast phantom and patient images in digital mammography and breast tomosynthesis," Med. Phys. 40(8), 081920 (2013).

[10] Hanley, J. A. and McNeil, B. J., "The meaning and use of the area under a receiver operating characteristic (ROC) curve," Radiology 143, 29-36 (1982). 